\title{
Electromagnetic corrections to leptonic pion decay from lattice QCD using infinite-volume reconstruction method
}

\section{Norman H. Christ}

Physics Department, Columbia University, New York, NY 10027, USA

E-mail: nhcephys.columbia.edu

\section{Xu Feng*}

School of Physics, Peking University, Beijing 100871, China,

Collaborative Innovation Center of Quantum Matter, Beijing 100871, China,

Center for High Energy Physics, Peking University, Beijing 100871, China,

State Key Laboratory of Nuclear Physics and Technology, Peking University, Beijing 100871,

China

E-mail: xu.feng@pku.edu.cn

\section{Lu-Chang Jin}

Department of Physics, University of Connecticut, Storrs, CT 06269, USA,

RIKEN-BNL Research Center, Brookhaven National Laboratory, Building 510, Upton, NY 11973

E-mail: ljin.luchang@gmail.com

\section{Christopher T. Sachrajda}

Department of Physics and Astronomy, University of Southampton, Southampton SO17 1BJ, UK

E-mail: ctsesoton.ac.uk

We present a lattice procedure to calculate the leptonic pion decay width with only exponentiallysuppressed finite-volume errors using the infinite-volume reconstruction method. In our approach, the infrared divergences from virtual-photon exchange and radiative emission can be cancelled in a simple, direct way using lattice hadronic functions as inputs.

37th International Symposium on Lattice Field Theory - Lattice2019

16-22 June 2019

Wuhan, China

\footnotetext{
* Speaker.
} 


\section{Introduction}

In the precision flavor physics where lattice QCD interplays with experimental measurements, the electromagnetic (EM) corrections become important when the lattice calculations reach the sub-percent precision. A milestone example is the pion and kaon leptonic decay constants. FLAG Review 2019 [1] reports the average of lattice results $f_{K} / f_{\pi}=1.1932(19)$. At this precision it is crucial to include the EM corrections.

Including the photon exchange in the lattice calculations usually causes the power-law finitevolume (FV) effects. In Ref. [2], a method called infinite-volume reconstruction (IVR) is developed to remove the power-law FV effects in the EM corrections to the hadron's mass. Later, this method has been successfully applied to the calculation of neutrinoless double beta decay [3]. In this work, we propose to use the IVR method for the lattice QCD calculation of the leptonic pion decay width.

\section{Leptonic pion decay in the infinite volume}
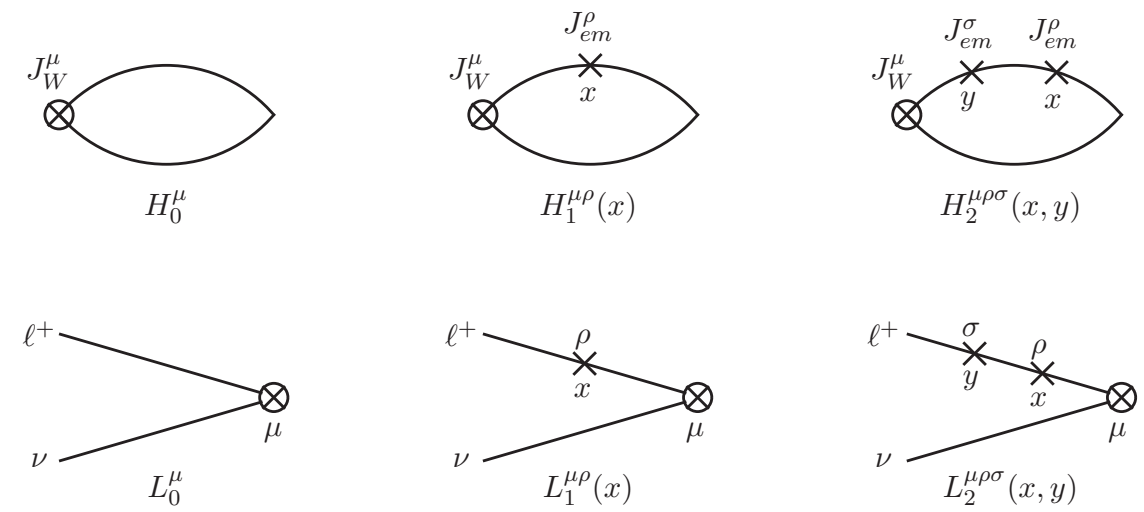

Figure 1: Samples of the hadronic and leptonic diagrams for $\pi \rightarrow \ell v(\gamma)$ decays.

With EM corrections, the leptonic pion decay amplitude shall be written as

$$
\begin{aligned}
\mathscr{A}[\pi \rightarrow \ell v] & =\left\langle\ell v\left|\mathscr{L}_{W}(0)\right| \pi\right\rangle+\frac{1}{2} \int d^{4} x \int d^{4} y\left\langle\ell v\left|T\left[\mathscr{L}_{e m}(x) \mathscr{L}_{e m}(y) \mathscr{L}_{W}(0)\right]\right| \pi\right\rangle, \\
\mathscr{A}[\pi \rightarrow \ell v \gamma] & =\int d^{4} x\left\langle\gamma \ell v\left|T\left[\mathscr{L}_{e m}(x) \mathscr{L}_{W}(0)\right]\right| \pi\right\rangle,
\end{aligned}
$$

where $\mathscr{L}_{W}=\frac{\sqrt{2}}{2} G_{F} V_{u d} J_{W}^{\mu} \bar{v} \gamma^{\mu}\left(1-\gamma_{5}\right) \ell$ is the standard, effective weak Lagrangian and $\mathscr{L}_{e m}=$ $e J_{e m}^{\mu} A^{\mu}+e \bar{\ell} \gamma^{\mu} \ell A^{\mu}$ is the EM one. Here $J_{W}^{\mu}$ and $J_{e m}^{\mu}$ are the relevant weak and EM quark currents. $G_{F}$ is the Fermi constant, $V_{u d}$ is the CKM matrix element and $e$ is the electric charge.

One can write the leptonic pion decay amplitude as the combination of a hadronic function, a leptonic function and a possible photon propagator or polarization vector. The hadronic function contains all the nonperturbative QCD information and shall be calculated using lattice QCD, while the leptonic one is analytically known. In the Fig. 1 we show the quark contractions for the hadronic diagrams in the upper panel. The relevant hadronic functions in the infinite volume are given by

$$
\begin{aligned}
H_{0}^{\mu} & =\left\langle 0\left|J_{W}^{\mu}(0)\right| \pi(\overrightarrow{0})\right\rangle, \\
H_{1}^{\mu \rho}(x) & =\left\langle 0\left|T\left[J_{e m}^{\rho}(x) J_{W}^{\mu}(0)\right]\right| \pi(\overrightarrow{0})\right\rangle, \\
H_{2}^{\mu \rho \sigma}(x, y) & =\left\langle 0\left|T\left[J_{e m}^{\rho}(x) J_{e m}^{\sigma}(y) J_{W}^{\mu}(0)\right]\right| \pi(\overrightarrow{0})\right\rangle,
\end{aligned}
$$


where $|\pi(\overrightarrow{0})\rangle$ indicates the initial pion state with zero spatial momentum. Another useful hadronic function is

$$
H_{2}^{\rho \sigma}(x)=\left\langle\pi(\overrightarrow{0})\left|T\left[J_{e m}^{\rho}(x) J_{e m}^{\sigma}(0)\right]\right| \pi(\overrightarrow{0})\right\rangle .
$$

It is related to the EM corrections to the hadron's mass and can be used in the analysis of the infrared (IR) divergence as we will show later.

The leptonic contractions are shown in the lower panel of Fig. 1. The relevant leptonic functions are given by

$$
\begin{aligned}
L_{0}^{\mu} & =\bar{u}\left(\vec{p}_{v}\right) \gamma^{\mu}\left(1-\gamma_{5}\right) v\left(\vec{p}_{\ell}\right), \\
L_{1}^{\mu \rho}(x) & =\bar{u}\left(\vec{p}_{v}\right) \gamma^{\mu}\left(1-\gamma_{5}\right) S_{\ell}(0, x) \gamma^{\sigma}, v\left(\vec{p}_{\ell}\right) e^{-i p_{\ell} x} \\
L_{2}^{\mu \rho \sigma}(x, y) & =\bar{u}\left(\vec{p}_{v}\right) \gamma^{\mu}\left(1-\gamma_{5}\right) S_{\ell}(0, x) \gamma^{\rho} S_{\ell}(x, y) \gamma_{\sigma} v\left(\vec{p}_{\ell}\right) e^{-i p_{\ell} y},
\end{aligned}
$$

with $S_{\ell}(x, y)$ the lepton's propagator.

Using the hadronic and leptonic functions, the amplitudes defined in Eq. (2.1) can be written as

$$
\mathscr{A}[\pi \rightarrow \ell v]=\frac{\sqrt{2}}{2} G_{F} V_{u d}\left(A_{0}+e^{2} A_{2}\right), \quad \mathscr{A}[\pi \rightarrow \ell v \gamma]=\frac{\sqrt{2}}{2} G_{F} V_{u d} e A_{1},
$$

with

$$
\begin{aligned}
& A_{0}=H_{0}^{\mu} L_{0}^{\mu}, \\
& A_{1}=\int d^{4} x\left[H_{1}^{\mu \rho}(x) L_{0}^{\mu}+H_{0}^{\mu} L_{1}^{\mu \rho}(x) e^{-i p_{\ell} x}\right] \varepsilon_{\lambda}^{\rho *}\left(p_{\gamma}\right) e^{-i p_{\gamma} x}, \\
& A_{2}=\frac{1}{2} \int d^{4} x \int d^{4} y\left[H_{0}^{\mu} L_{2}^{\mu \rho \sigma}(x, y)+H_{1}^{\mu \rho}(x) L_{1}^{\mu \sigma}(y)+H_{2}^{\mu \rho \sigma}(x, y) L_{0}^{\mu}\right] S_{\gamma}^{\rho \sigma}(x, y) .
\end{aligned}
$$

Here $\varepsilon_{\lambda}^{\rho *}\left(p_{\gamma}\right)$ is the photon's polarization vector with $\lambda$ the polarization direction. $S_{\gamma}^{\rho \sigma}(x, y)$ is the photon's propagator in Euclidean spacetime

$$
S_{\gamma}^{\rho \sigma}(x, y)=\int \frac{d^{4} p}{(2 \pi)^{4}} \frac{\hat{S}_{\gamma}^{\rho \sigma}(p)}{p^{2}} e^{i p(x-y)}=\int \frac{d^{3} \vec{p}}{(2 \pi)^{3}} \frac{\hat{S}_{\gamma}^{\rho \sigma}(i E, \vec{p})}{2 E} e^{-E\left|t_{x}-t_{y}\right|} e^{i \vec{p} \cdot(\vec{x}-\vec{y})}, \quad E=|\vec{p}| .
$$

The structure of $\hat{S}_{\gamma}^{\rho \sigma}$ is related to the gauge used for the photon's propagator. Summing over the polarization directions, we have

$$
\sum_{\lambda} \varepsilon_{\lambda}^{\rho *}\left(p_{\gamma}\right) \varepsilon_{\lambda}^{\sigma}\left(p_{\gamma}\right)=\hat{S}_{\gamma}^{\rho \sigma}\left(p_{\gamma}\right)
$$

The decay width for $\pi^{+} \rightarrow \ell^{+} v(\gamma)$ is given by

with

$$
\Gamma=\frac{1}{2} G_{F}^{2} V_{u d}^{2}\left(\Gamma^{(0 \gamma)}+\Gamma^{(1 \gamma)}\right)
$$

$$
\begin{aligned}
& \Gamma^{(0 \gamma)}=\int d \Phi_{2}\left(p_{\pi} ; p_{v}, p_{\ell}\right)\left\{\left|A_{0}\right|^{2}+2 e^{2} \operatorname{Re}\left[A_{0} A_{2}\right]\right\}, \\
& \Gamma^{(1 \gamma)}=\int d \Phi_{3}\left(p_{\pi} ; p_{v}, p_{\ell}, p_{\gamma}\right) e^{2}\left|A_{1}\right|^{2} .
\end{aligned}
$$

Here $d \Phi_{2}$ and $d \Phi_{3}$ indicate the 2-particle and 3-particle phase space.

It is worthwhile to mention that the calculation of amplitude $A_{2}$ is demanding as it requires the integral of $H_{2}^{\mu \rho \sigma}(x, y)$ over both $\vec{x}$ and $\vec{y}$ simultaneously. As a consequence the computational time scales as $L^{6}$ with $L$ the lattice size. Here we can simplify the integral as

$$
\int d^{4} x \int d^{4} y H_{2}^{\mu \rho \sigma}(x, y) L_{0}^{\mu} S_{\gamma}^{\rho \sigma}(x, y)=\int d^{4} x \int d t_{y} \hat{H}_{2}^{\mu \rho \sigma}\left(x ; t_{y}, \overrightarrow{0}\right) L_{0}^{\mu} S_{\gamma}^{\rho \sigma}\left(x ; t_{y}, \overrightarrow{0}\right)
$$


with

$$
\hat{H}_{2}^{\mu \rho \sigma}\left(x ; t_{y}, \overrightarrow{0}\right) \equiv \int d^{3} \vec{z}\left\langle 0\left|T\left[J_{e m}^{\rho}(x) J_{e m}^{\sigma}\left(t_{y}, \overrightarrow{0}\right) J_{W}^{\mu}(0,-\vec{z})\right]\right| \pi(\overrightarrow{0})\right\rangle
$$

When one generates the hadronic function $\hat{H}_{2}^{\mu \rho \sigma}\left(x ; t_{y}, \overrightarrow{0}\right)$, the integral over $\vec{z}$ can be performed in advance. Thus in right-hand-side of Eq. (2.11), only the spatial integral over $\vec{x}$ is required.

\section{Infinite-volume reconstruction method}

Now we move from the infinite-volume analysis in the previous section to a FV lattice calculation. The hadronic functions given in Eq. (2.2) shall be replaced by lattice quantities, which are specified by a subscript $L$ here

$$
H_{0}^{\mu}, H_{1}^{\mu \rho}(x), \hat{H}_{2}^{\mu \rho \sigma}\left(x ; t_{y}, \overrightarrow{0}\right) \Leftarrow H_{0, L}^{\mu}, H_{1, L}^{\mu \rho}(x), \hat{H}_{2, L}^{\mu \rho \sigma}\left(x ; t_{y}, \overrightarrow{0}\right) .
$$

Although the replacement of $H_{0}^{\mu}$ by $H_{0, L}^{\mu}$ only brings in exponentially-suppressed FV effects, such replacements for $H_{1}^{\mu \rho}(x)$ and $\hat{H}_{2}^{\mu \rho \sigma}\left(x ; t_{y}, \overrightarrow{0}\right)$ can cause the power-law FV effects as explained later. Therefore, we propose to use the IVR method [2] to remove these power-law effects.

3.1 $H_{1}^{\mu \rho}(x)$

Let us start with the case of $H_{1}^{\mu \rho}(x)$. At $t_{x}<0$, we have

$$
H_{1}^{\mu \rho}(x)=\left\langle 0\left|J_{W}^{\mu}(0) J_{e m}^{\rho}(x)\right| \pi(\overrightarrow{0})\right\rangle \sim e^{-m_{\pi} \sqrt{\bar{x}^{2}+t_{x}^{2}}} e^{-m_{\pi} t_{x}} .
$$

For large $\left|t_{x}\right|$, e.g. $\left|t_{x}\right| \gg|\vec{x}|, H_{1}^{\mu \rho}(x)$ scales as an $O(1)$ quantity. As a consequence, the replacement of $H_{1}^{\mu \rho}(x)$ by $H_{1, L}^{\mu \rho}(x)$ results in a power-law FV effect.

Following the method developed in Ref. [2], we choose an appropriate time $t_{s}\left(0<t_{s} \lesssim L\right)$, which ensures the ground intermediate state dominance in $H_{1}^{\mu \rho}(x)$. Namely, for $t \leq-t_{s}$ the hadronic function $H_{1}^{\mu \rho}(x)$ is given by

$$
H_{1}^{\mu \rho}(t, \vec{x}) \doteq \int \frac{d^{3} \vec{p}}{(2 \pi)^{3}}\left\langle 0\left|J_{W}^{\mu}(0)\right| \pi(\vec{p})\right\rangle \frac{1}{2 E_{\pi}}\left\langle\pi(\vec{p})\left|J_{e m}^{\rho}(0)\right| \pi(\overrightarrow{0})\right\rangle e^{\left(E_{\pi}-m_{\pi}\right) t} e^{-i \vec{p} \cdot \vec{x}},
$$

where the symbol $\doteq$ denotes the omission of the excited states. The spectral weight function can be determined using $H_{1}^{\mu \rho}\left(-t_{s}, \vec{x}\right)$ as an input

$$
\left\langle 0\left|J_{W}^{\mu}(0)\right| \pi(\vec{p})\right\rangle \frac{1}{2 E_{\pi}}\left\langle\pi(\vec{p})\left|J_{e m}^{\rho}(0)\right| \pi(\overrightarrow{0})\right\rangle \doteq \int d^{3} \vec{x} H_{1}^{\mu \rho}\left(-t_{s}, \vec{x}\right) e^{i \vec{p} \cdot \vec{x}} e^{\left(E_{\pi}-m_{\pi}\right) t_{s}} .
$$

Putting Eq. (3.4) into Eq. (3.3), we have

$$
H_{1}^{\mu \rho}(t, \vec{x})=\int d^{3} \vec{x}^{\prime} H_{1}^{\mu \rho}\left(-t_{s}, \vec{x}^{\prime}\right) \int \frac{d^{3} \vec{p}}{(2 \pi)^{3}} e^{i \vec{p} \cdot\left(\vec{x}^{\prime}-\vec{x}\right)} e^{\left(E_{\pi}-m_{\pi}\right)\left(t+t_{s}\right)} .
$$

It means that the hadronic function $H_{1}^{\mu \rho}(t, \vec{x})$ at $t \leq-t_{s}$ can be reconstructed using $H_{1}^{\mu \rho}\left(-t_{s}, \vec{x}\right)$. Therefore, the replacement can be performed through

$$
H_{1}^{\mu \rho}(t, \vec{x}) \Leftarrow H_{1}^{\mu \rho}\left(-t_{s}, \vec{x}\right) \Leftarrow H_{1, L}^{\mu \rho}\left(-t_{s}, \vec{x}\right) .
$$

As we have avoided using $H_{1, L}^{\mu \rho}(x)$ at large $|t|$, the replacement given in (3.6) only amounts for exponentially-suppressed FV effects. For more details, we refer the readers to Ref. [2]. 
3.2 $\hat{H}_{2}^{\mu \rho \sigma}\left(x ; t_{y}, \overrightarrow{0}\right)$

For $\hat{H}_{2}^{\mu \rho \sigma}\left(x ; t_{y}, \overrightarrow{0}\right)$ we assume $t_{x} \geq t_{y}$ without loss of generality. We classify the hadronic functions into three classes:

$$
I: t_{x} \geq t_{y}>0, \quad I I: t_{x} \geq 0 \geq t_{y}, \quad I I I: 0>t_{x} \geq t_{y} .
$$

For class $I$, we can split the hadronic function into two parts

$$
\hat{H}_{2}^{\mu \rho \sigma}\left(x ; t_{y}, \overrightarrow{0}\right)=\hat{H}_{2}^{\mu \rho \sigma,(0)}\left(x ; t_{y}, \overrightarrow{0}\right)+H_{2}^{\mu \rho \sigma,(1)}\left(x ; t_{y}, \overrightarrow{0}\right),
$$

with

$$
\begin{aligned}
& \hat{H}_{2}^{\mu \rho \sigma,(0)}\left(x ; t_{y}, \overrightarrow{0}\right)=\int d^{3} \vec{z}\left\langle 0\left|T\left[J_{e m}^{\rho}(x) J_{e m}^{\sigma}\left(t_{y}, \overrightarrow{0}\right)\right]\right| 0\right\rangle\left\langle 0\left|J_{W}^{\mu}(0)\right| \pi(\overrightarrow{0})\right\rangle, \\
& \hat{H}_{2}^{\mu \rho \sigma,(1)}\left(x ; t_{y}, \overrightarrow{0}\right)=\hat{H}_{2}^{\mu \rho \sigma}\left(x ; t_{y}, \overrightarrow{0}\right)-\hat{H}_{2}^{\mu \rho \sigma,(0)}\left(x ; t_{y}, \overrightarrow{0}\right) .
\end{aligned}
$$

The first term $\hat{H}_{2}^{\mu \rho \sigma,(0)}\left(x ; t_{y}, \overrightarrow{0}\right)$ is a vacuum contribution and shall be subtracted. The remaining contribution from $H_{2}^{\mu \rho \sigma,(1)}\left(x ; t_{y}, \overrightarrow{0}\right)$ is exponentially suppressed at large $|\vec{x}|$. Therefore, the replacement of $\hat{H}_{2}^{\mu \rho \sigma}\left(x ; t_{y}, \overrightarrow{\overrightarrow{0}}\right)$ by $\hat{H}_{2, L}^{\mu \rho \sigma}\left(x ; t_{y}, \overrightarrow{0}\right)$ only causes the exponentially-suppressed FV effects.

For class $I I$, the power-law FV effects could be introduced in the substitution of $\hat{H}_{2}^{\mu \rho \sigma}\left(x ; t_{y}, \overrightarrow{0}\right)$ using the lattice data at large $\left|t_{y}\right|$. So we make the replacement of

$$
\hat{H}_{2}^{\mu \rho \sigma}\left(x ; t_{y}, \overrightarrow{0}\right) \Leftarrow \hat{H}_{2}^{\mu \rho \sigma}\left(x ;-t_{s}, \overrightarrow{0}\right) \Leftarrow \hat{H}_{2, L}^{\mu \rho \sigma}\left(x ;-t_{s}, \overrightarrow{0}\right)
$$

for $t_{y} \leq-t_{s}$ and protect the lattice results from the power-law FV contamination.

For class $I I I$, when $t_{x}-t_{y} \geq t_{s}$, we make the replacement of

$$
\hat{H}_{2}^{\mu \rho \sigma}\left(x ; t_{y}, \overrightarrow{0}\right) \Leftarrow \hat{H}_{2}^{\mu \rho \sigma}\left(x ; t_{x}-t_{s}, \overrightarrow{0}\right) \Leftarrow \hat{H}_{2, L}^{\mu \rho \sigma}\left(x ; t_{x}-t_{s}, \overrightarrow{0}\right) .
$$

Again the hadronic function at large time separation $t_{x}-t_{y} \geq t_{s}$ is replaced by the one at modest time $t_{x}-t_{y}=t_{s}$, where $t_{s}$ is chosen to guarantee the ground-state dominance.

\section{Cancellation of infrared divergence}

It is known that the IR divergence cancels in the combination of the decay widths $\Gamma^{(0 \gamma)}$ and $\Gamma^{(1 \gamma)}$. In Ref. [4], two different IR regulators are used in $\Gamma^{(0 \gamma)}$ and $\Gamma^{(1 \gamma)}$, respectively. In the evaluation of $\Gamma^{(0 \gamma)}$, by using $\mathrm{QED}_{\mathrm{L}}$ technique the non-zero lattice momentum $p=2 \pi / L$ is used as an IR cutoff, while for $\Gamma^{(1 \gamma)}$ the nonzero photon mass is used as an IR regulator. The perturbative matching between the two different IR schemes is carried out under the assumption that the hadron's internal structure can be neglected in the IR limit and thus the hadron can be treated as a point-like particle. The pioneering work [5] is done following this direction.
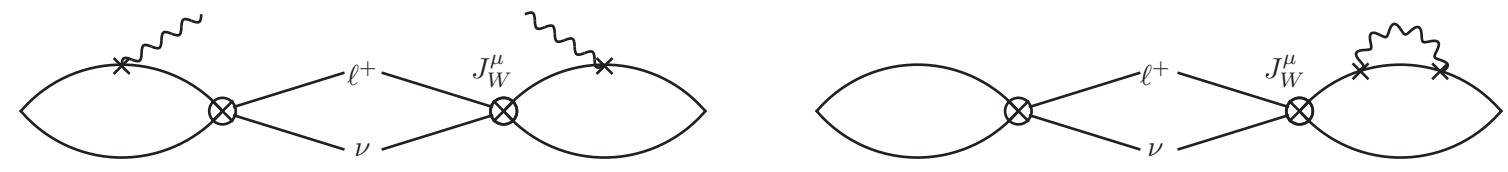

Figure 2: Samples of diagrams contributing to $\Gamma^{(1 \gamma)}$ (left) and $\Gamma^{(0 \gamma)}$ (right).

Using the IVR method, we will show that the IR divergence can be cancelled in a relatively simple way. Here we take Fig. 2 as an example. In the left panel, the decay width $\Gamma^{(1 \gamma)}$ receives the contribution from the amplitude square $|A|^{2}$ with $A$ defined as

$$
A=\int d^{4} x H_{1}^{\mu \rho}(x) L_{0}^{\mu} \varepsilon_{\lambda}^{\rho *} e^{-i p_{\gamma} x} .
$$


We concentrate on the integral region $t \in\left(\infty,-t_{s}\right]$ and have the amplitude defined as

$$
\hat{A} \equiv \int_{-\infty}^{-t_{s}} d t \int d^{3} \vec{x} H_{1}^{\mu \rho}(t, \vec{x}) L_{0}^{\mu} \varepsilon_{\lambda}^{\rho *} e^{E_{\gamma} t} e^{-i \vec{p}_{\gamma} \cdot \vec{x}} \doteq \tilde{H}_{1}^{\mu \rho}\left(-t_{s},-\vec{p}_{\gamma}\right) L_{0}^{\mu} \varepsilon_{\lambda}^{\rho *} \frac{e^{-E_{\gamma} t_{s}}}{E_{\pi}+E_{\gamma}-m_{\pi}},
$$

with

$$
\tilde{H}_{1}^{\mu \rho}\left(-t_{s},-\vec{p}_{\gamma}\right)=\int d^{3} \vec{x} H_{1}^{\mu \rho}\left(-t_{s}, \vec{x}\right) e^{-i \vec{p}_{\gamma} \cdot \vec{x}}
$$

For $\vec{p}_{\gamma}=0$, we have $\tilde{H}_{1}^{\mu \rho}\left(-t_{s}, \overrightarrow{0}\right) \doteq H_{0}^{\mu} \delta^{\rho 0}$.

We can split $|\hat{A}|^{2}$ into two terms

$$
|\hat{A}|^{2} \doteq|\hat{A}|_{\text {con }}^{2}+|\hat{A}|_{\text {div }}^{2}
$$

with the definitions

$$
\begin{aligned}
|\hat{A}|_{\text {con }}^{2} & =\int d^{3} \vec{x} \int d^{3} \vec{y} H_{1}^{\mu \rho}\left(-t_{s}, \vec{x}\right) H_{1}^{\mu^{\prime} \rho^{\prime} *}\left(-t_{s}, \vec{y}\right)\left(e^{-i \vec{p}_{\gamma} \cdot(\vec{x}-\vec{y})}-1\right) L_{0}^{\mu} L_{0}^{\mu^{\prime} *} \varepsilon^{\rho *} \varepsilon^{\rho^{\prime}} \frac{e^{-2 E_{\gamma} t_{s}}}{\left(E_{\pi}+E_{\gamma}-m_{\pi}\right)^{2}}, \\
|\hat{A}|_{\text {div }}^{2} & \doteq\left|H_{0}^{\mu} L_{0}^{\mu}\right|^{2} \varepsilon^{0 *} \varepsilon^{0} \frac{e^{-2 E_{\gamma} t_{s}}}{\left(E_{\pi}+E_{\gamma}-m_{\pi}\right)^{2}} .
\end{aligned}
$$

Note that $|\hat{A}|_{\text {div }}^{2}$ is proportional to $1 /\left|\vec{p}_{\gamma}\right|^{2}$ and the phase space $d \Phi_{3}$ is proportional to $\frac{1}{(2 \pi)^{3}} \frac{d^{3} \vec{p}_{\gamma}}{2\left|\vec{p}_{\gamma}\right|}$. Thus $|\hat{A}|_{\text {div }}^{2}$ causes an IR divergence to $\Gamma^{(1 \gamma)}$, while the contributions from $|\hat{A}|_{\text {con }}^{2}$ are IR convergent due to the suppression of the factor $e^{-i \vec{p}_{\gamma}(\vec{x}-\vec{y})}-1$.

As a next step, we examine the IR structure in the right panel of Fig. 2, where the decay width $\Gamma^{(0 \gamma)}$ receives the contribution from $2 \operatorname{Re}\left[B B_{0}^{*}\right]$, with

$$
B \equiv \frac{1}{2} \int d^{4} x \int d t_{y} \hat{H}_{2}^{\mu \rho \sigma}\left(x ; t_{y}, \overrightarrow{0}\right) L_{0}^{\mu} S_{\gamma}^{\rho \sigma}\left(x ; t_{y}, \overrightarrow{0}\right), \quad B_{0} \equiv H_{0}^{\mu} L_{0}^{\mu} .
$$

We concentrate on the integral regions of $-T \leq t_{x} \leq-t_{s}$ and $-T \leq t_{y} \leq t_{x}-t_{s}$, and define the relevant contribution as $\hat{B}$

$$
\hat{B} \equiv \frac{1}{2} \int_{-T}^{-t_{s}} d t_{x} \int_{-T}^{t_{x}-t_{s}} d t_{y} \int d^{3} \vec{x} \hat{H}_{2}^{\mu \rho \sigma}\left(x ; t_{y}, \overrightarrow{0}\right) L_{0}^{\mu} S_{\gamma}^{\rho \sigma}\left(x ; t_{y}, \overrightarrow{0}\right) .
$$

Again, here $t_{s}$ is chosen to guarantee the ground state dominance. After performing the integral, we have

$$
\begin{aligned}
\hat{B} \doteq \frac{1}{2} \int \frac{d^{3} \vec{p}}{(2 \pi)^{3}} \frac{H_{0}^{\mu}}{2 m_{\pi}}\left\langle\pi(\overrightarrow{0})\left|J_{e m}^{\rho}(0)\right| \pi(\vec{p})\right\rangle \frac{1}{2 E_{\pi}}\left\langle\pi(\vec{p})\left|J_{e m}^{\sigma}(0)\right| \pi(\overrightarrow{0})\right\rangle L_{0}^{\mu} \frac{\hat{S}_{\gamma}^{\rho \sigma}\left(p_{\gamma}\right)}{2 E_{\gamma}} \\
\left(\frac{e^{-\left(E_{\pi}+E_{\gamma}-m_{\pi}\right) t_{s}}}{E_{\pi}+E_{\gamma}-m_{\pi}}\left(T-t_{s}\right)+\frac{e^{-\left(E_{\pi}+E_{\gamma}-m_{\pi}\right)\left(T-t_{s}\right)}-1}{\left(E_{\pi}+E_{\gamma}-m_{\pi}\right)^{2}}\right),
\end{aligned}
$$

with $p_{\gamma}=\left(i E_{\gamma},-\vec{p}\right)$ and $E_{\gamma}=|\vec{p}|$. In Eq. (4.8) the first term in the parenthesis is relevant for the EM correction to the hadron's mass and the second term causes the IR divergence as it contains a factor of $\frac{1}{2 E_{\gamma}} \frac{1}{\left(E_{\pi}+E_{\gamma}-m_{\pi}\right)^{2}}$. In the IVR method, the hadronic matrix element in Eq. (4.8) can be constructed using $H_{2}^{\rho \sigma}\left(t_{s}, \vec{x}\right)$ as an input

$$
\left\langle\pi(\overrightarrow{0})\left|J_{e m}^{\rho}(0)\right| \pi(\vec{p})\right\rangle \frac{1}{2 E_{\pi}}\left\langle\pi(\vec{p})\left|J_{e m}^{\sigma}(0)\right| \pi(\overrightarrow{0})\right\rangle \doteq \int d^{3} \vec{x} H_{2}^{\rho \sigma}\left(t_{s}, \vec{x}\right) e^{-i \vec{p} \cdot \vec{x}} e^{\left(E_{\pi}-m_{\pi}\right) t_{s}}
$$

Here we focus on the IR divergent contribution, namely

$$
\bar{B} \equiv-\frac{1}{2} \int \frac{d^{3} \vec{p}}{(2 \pi)^{3}} \frac{H_{0}^{\mu}}{2 m_{\pi}} \int d^{3} \vec{x} H_{2}^{\rho \sigma}\left(t_{s}, \vec{x}\right) e^{-i \vec{p} \cdot \vec{x}} L_{0}^{\mu} \frac{\hat{S}_{\gamma}^{\rho \sigma}\left(p_{\gamma}\right)}{2 E_{\gamma}} \frac{e^{\left(E_{\pi}-m_{\pi}\right) t_{s}}}{\left(E_{\pi}+E_{\gamma}-m_{\pi}\right)^{2}} .
$$


Again, we split $\bar{B}_{2}$ into two terms

$$
\bar{B}=\bar{B}_{\text {con }}+\bar{B}_{\text {div }}
$$

with

$$
\begin{aligned}
\bar{B}_{\mathrm{con}} & =-\frac{1}{2} \int \frac{d^{3} \vec{p}}{(2 \pi)^{3}} \frac{H_{0}^{\mu}}{2 m_{\pi}} \int d^{3} \vec{x} H_{2}^{\rho \sigma}\left(t_{s}, \vec{x}\right)\left(e^{-i \vec{p} \cdot \vec{x}}-1\right) L_{0}^{\mu} \frac{\hat{S}_{\gamma}^{\rho \sigma}\left(p_{\gamma}\right)}{2 E_{\gamma}} \frac{e^{\left(E_{\pi}-m_{\pi}\right) t_{s}}}{\left(E_{\pi}+E_{\gamma}-m_{\pi}\right)^{2}}, \\
\bar{B}_{\mathrm{div}} & =-\frac{1}{2} \int \frac{d^{3} \vec{p}}{(2 \pi)^{3}} H_{0}^{\mu} L_{0}^{\mu} \frac{\hat{S}_{\gamma}^{00}\left(p_{\gamma}\right)}{2 E_{\gamma}} \frac{e^{\left(E_{\pi}-m_{\pi}\right) t_{s}}}{\left(E_{\pi}+E_{\gamma}-m_{\pi}\right)^{2}} .
\end{aligned}
$$

Here only $\bar{B}_{\text {div }}$ causes the IR divergence to $\Gamma^{(0 \gamma)}$.

We can combine the $|\hat{A}|_{\text {div }}^{2}$ contributions to $\Gamma^{(1 \gamma)}$ and $\bar{B}_{\text {div }}$ contributions to $\Gamma^{(0 \gamma)}$ and obtain

$$
\begin{aligned}
I & =\int d \Phi_{3}\left(p_{\pi} ; p_{v}, p_{\ell}, p_{\gamma}\right)|\hat{A}|_{\mathrm{div}}^{2}+\int d \Phi_{2}\left(p_{\pi} ; p_{v}, p_{\ell}\right) 2 \operatorname{Re}\left[\bar{B}_{\mathrm{div}} B_{0}^{*}\right] \\
& =\int \frac{d^{3} \vec{p}_{\gamma}}{(2 \pi)^{3}} \frac{\hat{S}_{\gamma}^{00}\left(p_{\gamma}\right)}{2 E_{\gamma}} \frac{1}{\left(E_{\pi}+E_{\gamma}-m_{\pi}\right)^{2}}\left[e^{-2 E_{\gamma} t_{s}} f\left(\left(p_{\pi}-p_{\gamma}\right)^{2}\right)-e^{\left(E_{\pi}-m_{\pi}\right) t_{s}} f\left(p_{\pi}^{2}\right)\right],
\end{aligned}
$$

where the function $f\left(p^{2}\right)$ is defined as

$$
f\left(p^{2}\right) \equiv \int d \Phi_{2}\left(p ; p_{v}, p_{\ell}\right)\left|H_{0}^{\mu} L_{0}^{\mu}\right|^{2} .
$$

Here we have used the recursion relation $\int d \Phi_{3}\left(p_{\pi} ; p_{v}, p_{\ell}, p_{\gamma}\right)=\int \frac{d^{3} \vec{p}_{\gamma}}{(2 \pi)^{3}} \frac{1}{2 E_{\gamma}} \int d \Phi_{2}\left(p_{\pi}-p_{\gamma} ; p_{v}, p_{\ell}\right)$. Note that in Eq. (4.13) the factor in the bracket vanishes in the limit of $\left|\vec{p}_{\gamma}\right| \rightarrow 0$. Thus the result of $I$ is IR finite. The calculation of $I$ is simple, as it only requires the lattice QCD inputs of $H_{0}^{\mu}$.

\section{Conclusion}

In this work, we propose to calculate the leptonic pion decay width using the IVR method. We give the key ingredients to explain how the power-law FV effects are removed and how the IR divergence are cancelled in a simple way. In the upcoming work, we will also include the analysis of the wave function renormalization using the IVR method. We believe that the theoretical foundation is set up for a realistic lattice calculation of the leptonic pion decay width without powerlaw FV errors.

\section{References}

[1] Flavour Lattice Averaging Group collaboration, FLAG Review 2019, 1902.08191.

[2] X. Feng and L. Jin, QED self energies from lattice QCD without power-law finite-volume errors, Phys. Rev. D100 (2019) 094509 [1812.09817].

[3] X.-Y. Tuo, X. Feng and L.-C. Jin, Long-distance contributions to neutrinoless double beta decay $\pi^{-} \rightarrow \pi^{+} e e$, Phys. Rev. D100 (2019) 094511 [1909.13525].

[4] N. Carrasco, V. Lubicz, G. Martinelli, C. T. Sachrajda, N. Tantalo, C. Tarantino et al., QED Corrections to Hadronic Processes in Lattice QCD, Phys. Rev. D91 (2015) 074506 [1502 . 00257].

[5] D. Giusti, V. Lubicz, G. Martinelli, C. T. Sachrajda, F. Sanfilippo, S. Simula et al., First lattice calculation of the QED corrections to leptonic decay rates, Phys. Rev. Lett. 120 (2018) 072001 [1711.06537]. 